Classification

Physics Abstracts

$42.65-42.80$

\title{
Critical exponents in dispersive optical bistability
}

\author{
G. Grynberg and S. Cribier \\ Laboratoire de Spectroscopie Hertzienne de l'E.N.S., \\ Université Pierre et Marie Curie, 75230 Paris Cedex 05, France
}

(Reçu le 29 décembre 1982, révisé le 28 mars 1983, accepté le 19 avril 1983)

\begin{abstract}
Résumé. - On étudie théoriquement le temps de basculement $t_{\mathrm{s}}$ d'un système optique dont la bistabilité est liée à un effet de dispersion non linéaire. On montre que, si on fait varier un paramètre extérieur $p$ (intensité incidente ou longueur du Fabry-Perot) au-delà du point critique $p_{\mathrm{c}}, t_{\mathrm{s}}$ varie comme $\left(p-p_{\mathrm{c}}\right)^{-1 / 2}$ quelle que soit l'origine de la dispersion non linéaire.

Abstract. - We theoretically investigate the switching time $t_{\mathrm{s}}$ of a single beam optical device whose bistability relies on a dispersive effect. We show that if we vary an external parameter $p$ (incident intensity or length of the Fabry-Perot cavity) beyond a critical point $p_{\mathrm{c}}, t_{\mathrm{s}}$ should vary as $\left(p-p_{\mathrm{c}}\right)^{-1 / 2}$ whatever the origin of the non linear dispersion is.
\end{abstract}

The knowledge of transient behaviour in optical bistability is very important for technical purposes. Furthermore, the variation of the switching time as a function of a parameter can have a general physical signification. For instance, it is well known that the switching time goes to infinity when the bistable system is close to the critical point (" critical slowing down »). This effect is theoretically known [1,2] and has been observed for hybrid devices [3]. Similar effects have been discussed in other fields of physics [4, 5]. It is clearly important to know the variation of the switching time for values of parameters close to the critical ones. In a preceding paper [6], we have analysed the case of dispersive optical bistability in a situation where the non linear refractive index $n_{\mathrm{NL}}$ is a linear function of the intensity. We have shown that the switching time $t_{\mathrm{s}}$ should vary as the inverse of the square root of the difference between $p$ and $p_{\mathrm{c}}$ where $p$ is an external parameter characterizing the system. In the present paper, we want to show that this result has a much broader signification since it is valid whatever the law of variation of the non linear refractive index $n_{\mathrm{NL}}(I)$. In particular, it permits to understand the transients in two-photon optical bistability [7] in a situation where the linear law for the refractive index does not hold [8].

For a Fabry-Perot with two plane mirrors of reflectivity $R$ and transmission $T(R+T=1)$, the transmission $\theta$ is equal to :

$$
\theta(\Phi)=\frac{1}{1+\frac{4 R}{(1-R)^{2}} \sin ^{2} \Phi}
$$

(in the following, we will suppose that the reflectivity $R$ is close to 1 ). We assume that the FabryPerot is filled with a medium whose refractive index depends on the value of the intensity $I_{i}$ 
inside the Fabry-Perot. At equilibrium, the phase of light after one trip through the Fabry-Perot is equal to :

$$
\Phi=\delta+\Phi_{\mathrm{NL}},
$$

where $\delta$ is the value of $\Phi$ when the intensity goes to zero. In the case of a plane Fabry-Perot, we have $\delta=2 \pi n_{0} \frac{l}{\lambda}$, where $n_{0}$ is the usual refractive index, $l$ and $\lambda$ are the length of the cavity and the wavelength of light in the vacuum. $\Phi_{\mathrm{NL}}$ is equal to $2 \pi n_{\mathrm{NL}} \frac{l}{\lambda}$ where $n_{\mathrm{NL}}$ is the non linear refractive index. For example, for a two-level atom, $n_{\mathrm{NL}}$ is equal to [9] :

$$
n_{\mathrm{NL}}=n_{0}-\frac{N}{2 \varepsilon_{0}} \frac{d^{4} I_{i} \Gamma_{\mathrm{eg}} / \Gamma_{\mathrm{e}}}{\delta E\left(\delta E^{2}+\frac{\Gamma_{\mathrm{eg}}}{\Gamma_{\mathrm{e}}} d^{2} I_{i}\right)},
$$

if the detuning $\delta E$ from the resonance is large compared to $\hbar \Gamma_{\mathrm{eg}} . N$ is the number of atoms per unit of volume, $d$ is the matrix element of the electric dipole moment between $\mathrm{g}$ and e, $\Gamma_{\mathrm{eg}}$ and $\Gamma_{\mathrm{e}}$ are the relaxation rates of the optical coherence and of the population of the excited level.

In the case of a two-photon transition for a two-level atom, $n_{\mathrm{NL}}$ is equal to [10] :

$$
n_{\mathrm{NL}}=n_{0}+\frac{N}{2 \varepsilon_{0}} \frac{q^{2} I_{i}\left(\delta E-2 s \Gamma_{\mathrm{eg}} / \Gamma_{\mathrm{e}}\right)}{\left(\delta E^{2}+\hbar^{2} \Gamma_{\mathrm{eg}}^{2}+\frac{\Gamma_{\mathrm{eg}}}{\Gamma_{\mathrm{e}}} q^{2} I_{i}^{2}\right)},
$$

where $q$ is the matrix element of the two-photon operator $Q$ [11] between $g$ and e and $s$ is the dynamic Stark effect of the g-e transition. In particular, it can be noticed that at resonance, the refractive index varies as $I_{i}^{2}$ for small values of $I_{i}$.

In any case, since $I_{i}$ is proportional to $\theta I, I_{i}$, and therefore $\Phi_{\mathrm{NL}}$, can be written as functions of $\Phi$ and $I$. At equilibrium, we have :

$$
\Phi_{\mathrm{NL}}=f(I, \Phi) \text {. }
$$

We first consider the case when the time constant associated with the cavity is small compared to the one related to the medium. In this case (which corresponds to our experimental situation [7]) the equation (2) is true at any moment and the dynamics is determined by the equation :

$$
\frac{1}{\Gamma} \frac{\mathrm{d}}{\mathrm{d} t} \Phi+[\Phi-\delta-f(I, \Phi)]=0
$$

$\Gamma$ is a typical time constant for the atomic vapour. $\Gamma$ is generally of the same order as $\Gamma_{\mathrm{e}}$, even in the case of non resonant excitation because the excited level can be really populated by the collisions [12]. $\Gamma_{\mathrm{e}}$ may also be different from the radiative lifetime of the isolated atom because of the trapping of radiation.

We now discuss the properties of this equation when the parameters are close to the critical point. We first consider the case where $I$ is constant $[8,13]$. The coordinates of the critical point are $\Phi_{c}$ and $\delta_{c}$. They verify (see Fig. 1) :

$$
\begin{aligned}
& \delta_{\mathrm{c}}=\Phi_{\mathrm{c}}-f\left(I, \Phi_{\mathrm{c}}\right), \\
& \frac{\mathrm{d} \delta}{\mathrm{d} \Phi}=0 \Rightarrow \frac{\partial}{\partial \Phi} f\left(I, \Phi_{\mathrm{c}}\right)=1 .
\end{aligned}
$$




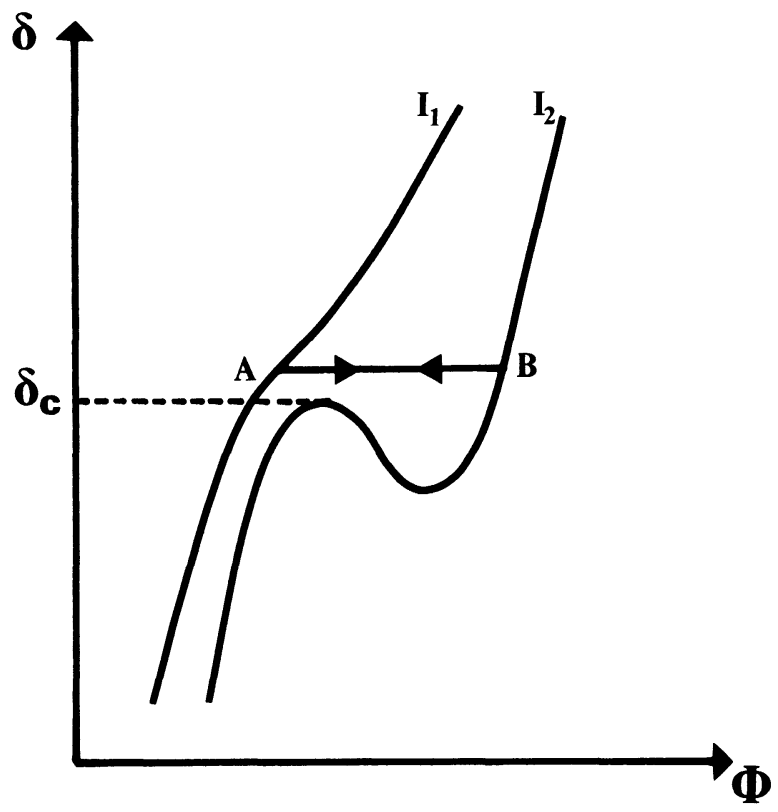

Fig. 1. - The static solution of the problem are given by $\Phi-\delta-f(I, \Phi)=0$ (see Eq. (6)). We have plot here two curves corresponding to different values of $I\left(I_{1}\right.$ and $\left.I_{2}\right)$. In actual experiments [7, 16], we make sudden changes of incident intensity from $I_{1}$ to $I_{2}$ and then from $I_{2}$ to $I_{1}$. In particular, transients corresponding to a change from the lower part of the bistability curve to the upper part are connected with paths like AB. We have calculated in this paper an order of magnitude of the switching time between $A$ and $B$ for values of $\delta$ close to $\delta_{\mathrm{c}}$. Even if the initial value of $\Phi$ differs from different values of $\delta$, the " critical slowing down " only occurs for a small range of values of $\delta$ (close to $\delta_{c}$ ) where the initial value of the $\operatorname{Arctg}$ (Eq. 10) should not vary much in the general case.

For coordinates close to those of the critical point, we develop equation (6) :

$$
\frac{1}{\Gamma} \frac{\mathrm{d} \Phi}{\mathrm{d} t}-\frac{1}{2} f_{\Phi}^{\prime \prime}\left(\Phi-\Phi_{\mathrm{c}}\right)^{2}-\left(\delta-\delta_{\mathrm{c}}\right)=0
$$

where

$$
f_{\Phi}^{\prime \prime}=\frac{\partial^{2} f}{\partial \Phi^{2}}\left(I, \Phi_{\mathrm{c}}\right)
$$

This equation has no static solution because $\left(\delta-\delta_{\mathrm{c}}\right)$ and $f_{\Phi}^{\prime \prime}$ have the same sign (we can verify in Fig. 1 that the transients involving $\delta>\delta_{\mathrm{c}}$ correspond to $\mathrm{d}^{2} \delta / \mathrm{d} \Phi^{2}<0$ and then $\mathrm{f}_{\Phi}^{\prime \prime}>0$. On the other hand, $\delta<\delta_{\mathrm{c}}$ corresponds to $f_{\Phi}^{\prime \prime}<0$ ).

This equation can be easily integrated :

$$
\operatorname{Arctg}\left[\sqrt{\frac{f_{\Phi}^{\prime \prime}}{2}} \frac{\left(\Phi-\Phi_{c}\right)}{\left(\delta-\delta_{c}\right)^{1 / 2}}\right]=\Gamma \sqrt{\frac{f_{\Phi}^{\prime \prime}}{2}}\left(\delta-\delta_{c}\right)^{1 / 2} t .
$$

The left side of equation (10) is of the order of $\pi$ and one can thus estimate the scaling law for the switching time $t_{\mathrm{s}}$ :

$$
t_{\mathrm{s}} \sim \frac{1}{\Gamma \sqrt{f_{\phi}^{\prime \prime}}}\left|\delta-\delta_{\mathrm{c}}\right|^{-1 / 2}
$$


The law of variation of the switching time as a function of the shift from the critical point is thus independent of the exact dependence of the refractive index upon the intensity. In dispersive optical bistability, one should find the same square root dependence in the case of one-photon [13, 14] or two-photon [8] optical bistability for large or small saturation.

We can derive a similar result for transients performed for a given value of $\delta$ by varying the intensity $[14,15]$. In that case, the coordinates of the critical points are $\phi_{\mathrm{c}}$ and $I_{\mathrm{c}}$. Instead for equation (9), we have :

$$
\frac{1}{\Gamma} \frac{\mathrm{d} \phi}{\mathrm{d} t}-\frac{1}{2} f_{\phi}^{\prime \prime}\left(\phi-\phi_{\mathrm{c}}\right)^{2}-f_{I}^{\prime}\left(I-I_{\mathrm{c}}\right)=0
$$

where

$$
f_{\phi}^{\prime \prime}=\frac{\partial^{2} f}{\partial \phi^{2}}\left(I_{\mathrm{c}}, \phi_{\mathrm{c}}\right) \quad \text { and } \quad f_{I}^{\prime}=\frac{\partial f}{\partial I}\left(I_{\mathrm{c}}, \phi_{\mathrm{c}}\right)
$$

This equation is similar to equation (9), so we find that the switching time in this case is of the order of :

$$
t_{\mathrm{s}} \sim \frac{1}{\Gamma \sqrt{\left|f_{\phi}^{\prime \prime} f_{I}^{\prime}\right|}}\left|I-I_{\mathrm{c}}\right|^{-1 / 2} .
$$

Equations (11) and (13) show that in each case, the switching time varies as $\left(p-p_{\mathrm{c}}\right)^{-1 / 2}$ where $p$ is the externally changed parameter. The transformation of $(6)$ into the approximate differential equations (9) or (12) is of course only true for small values of $\left(p-p_{c}\right)$ and $\left(\phi-\phi_{c}\right)$. If $\left(p-p_{c}\right)$ is a constant during the transient, $\left(\phi-\phi_{c}\right)$ varies and its value can be large at the beginning and at the end of the transient. However, the major part of the switching time comes from the points where $\left(\phi-\phi_{c}\right)$ is small and one expects that the real switching time will generally be of the same order of magnitude as the one calculated with this approximation.

Up to now we have considered the case where the time constant associated with the cavity is short compared to the time constant associated with the medium. However the theory can be extended for other situations. Let us assume that $x$ and $y$ are quantities describing the cavity and the medium and that they obey the following equations :

$$
\begin{aligned}
& \frac{\mathrm{d}}{\mathrm{d} t} x=-\gamma[x-g(y, p)], \\
& \frac{\mathrm{d}}{\mathrm{d} t} y=-\Gamma[y-f(x, p)],
\end{aligned}
$$

$\gamma^{-1}$ is the typical damping time of the cavity. We note $\tilde{x}=x-x_{\mathrm{c}}, \tilde{y}=y-y_{\mathrm{c}}$ and we develop (14) and (15) around the critical point :

$$
\begin{aligned}
& \frac{\mathrm{d}}{\mathrm{d} t} \tilde{x}=-\gamma\left[\tilde{x}-g_{y}^{\prime} \tilde{y}-g_{p}^{\prime}\left(p-p_{\mathrm{c}}\right)-\frac{1}{2} g_{y}^{\prime \prime} \tilde{y}^{2}\right] \\
& \frac{\mathrm{d}}{\mathrm{d} t} \tilde{y}=-\Gamma\left[\tilde{y}-f_{x}^{\prime} \tilde{x}-f_{p}^{\prime}\left(p-p_{\mathrm{c}}\right)-\frac{1}{2} f_{x}^{\prime \prime} \tilde{x}^{2}\right] .
\end{aligned}
$$

At the critical point, we have $f_{x}^{\prime} g_{y}^{\prime}=1$.

We take the new variables

$$
\begin{aligned}
\tau & =(\gamma+\Gamma) t \\
X & =(\gamma+\Gamma)\left[\frac{\tilde{x}}{\gamma}+g_{y}^{\prime} \frac{\tilde{y}}{\Gamma}\right], \\
Y & =\tilde{x}-g_{y}^{\prime} \tilde{y}
\end{aligned}
$$


We obtain the following equations for $X$ and $Y$

$$
\begin{aligned}
& \frac{\mathrm{d}}{\mathrm{d} \tau} Y=-Y+0\left(p-p_{\mathrm{c}}, X^{2}, Y^{2}\right), \\
& \frac{\mathrm{d}}{\mathrm{d} \tau} X=\frac{\gamma^{2} \Gamma^{2}}{(\gamma+\Gamma)^{4}} \frac{X^{2}}{2}\left[\frac{g_{y}^{\prime \prime}}{g_{y}^{\prime 2}}+f_{x}^{\prime \prime} g_{y}^{\prime}\right]+\left(p-p_{\mathrm{c}}\right)\left[g_{p}^{\prime}+g_{y}^{\prime} f_{p}^{\prime}\right]+0\left(Y^{2}, X Y\right) .
\end{aligned}
$$

Thus after a time of the order of unity, $Y$ reaches a value which is 0 at the precision of our development. The dynamics is then determined by equation (22) where we neglect the term in $0\left(Y^{2}, X Y\right)$. Equation (22) can be transformed into :

$$
\frac{\mathrm{d}}{\mathrm{d} \tau} X=\left[g_{p}^{\prime}+g_{y}^{\prime} f_{p}^{\prime}\right]\left[-\frac{\gamma^{2} \Gamma^{2}}{(\Gamma+\gamma)^{4}}\left(\frac{\mathrm{d}^{2} p}{\mathrm{~d} x^{2}}\right)_{\mathrm{c}} \frac{X^{2}}{2}+\left(p-p_{\mathrm{c}}\right)\right] .
$$

This equation is similar to (9) and leads to a switching time of the order of :

$$
t_{\mathrm{s}} \sim \frac{\Gamma+\gamma}{\Gamma \gamma} \frac{\left[-\left(\frac{\mathrm{d}^{2} p}{\mathrm{~d} x^{2}}\right)_{\mathrm{c}}\left(p-p_{\mathrm{c}}\right)\right]^{-1 / 2}}{g_{p}^{\prime}+g_{y}^{\prime} f_{p}^{\prime}} .
$$

In conclusion, we have shown that the switching time in single beam dispersive optical bistability should exhibit the same law of variation whatever the physical origin of the non linear dispersion is. New experiments [16] are in progress in order to verify the theory.

\section{Acknowledgments.}

We are very indebted to Dr. D. Delande and Dr. F. Biraben for very helpful conversations about this subject.

\section{References}

[1] Bonifacio, R. and Lugiato, L., Opt. Commun. 19 (1976) 172; Phys. Rev. A 18 (1978) 112.

[2] Bonifacio, R. and Meystre, P., Opt. Commun. 29 (1979) 131.

[3] Garmire, E., Marburger, J. H., Allen, S. D. and Winful, H. G., App. Phys. Lett. 34 (1979) 374.

[4] Martin, P. C., J. Physique Colloq. 37 (1976) C1-57.

[5] Puech, L., Hebral, B., Thoulouze, D. et Castaing, B., J. Physique Lett. 44 (1983) L-159.

[6] Grynberg, G., Biraben, F. and Giacobino, E., App. Phys. B 26 (1981) 155.

[7] Giacobino, E., Cribier, S., Grynberg, G. and Biraben, F., App. Phys. B 28 (1982) 170.

[8] Giacobino, E., Devaud, M., Biraben, F. and Grynberg, G., Phys. Rev. Lett. 45 (1980) 434.

[9] Sargent III, M., Scully, M. O. and Lamb Jr, W. E., Laser Physics (Addison-Wesley Publishing Company) 1974.

[10] Grynberg, G., Devaud, M., Flytzanis, C. and Cagnac, B., J. Physique 41 (1980) 931.

[11] Grynberg, G. and Cagnac, B., Rep. Prog. Phys. 40 (1977) 791.

[12] Carlsten, J. L. and SzüKe, A., J. Phys. B 9 (1976) L-231.

[13] Sandle, W. J. and Gallagher, A., Phys. Rev. A 24 (1981) 2017.

[14] Gibbs, H. M., Mac Call, S. L., Venkatesan, T. N. C., Phys. Rev. Lett. 36 (1976) 1135.

[15] Bischofberger, T. and Shen, Y. R., Phys. Rev. A 19 (1979) 1169.

[16] Cribier, S., Giacobino, E. and Grynberg, G., to be published. 